\title{
Serotonin Modulates Early Cortical Auditory Processing in Healthy Subjects. Evidence from MEG with Acute Tryptophan Depletion
}

Seppo Kähkönen, Jyrki Ahveninen, Sirpa Pennanen, Jyrki Liesivuori, Risto J. Ilmoniemi, and Iiro P. Jääskeläinen

We studied the effects of acute tryptophan depletion (ATD) on early cortical auditory processing. Middle-latency auditory evoked fields (MAEF) were investigated in 14 healthy subjects after $5 \mathrm{~h}$ of ATD or control mixture ingestion in a randomized, double-blinded, controlled crossover design study. MAEFs to monaural click stimuli (0.1ms duration) were recorded with a 122-channel neuromagnetometer. Total plasma tryptophan (Trp), free Trp, and large neutral amino acid (LNAA) concentrations were determined by using high-performance liquid chromatography. ATD lowered the total plasma Trp levels by $75 \%$, free Trp level by $47 \%$, and the ratio Trp/ $\Sigma L N A A$ by $92 \%$. The control mixture increased total Trp level by $45 \%$ and free Trp by $32 \%$, and decreased the ratio Trp/

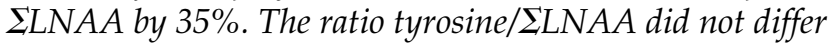

between ATD and control experiment. ATD resulted in a significant main effect on Pam latencies and a nearsignificant main effect on Pam amplitudes. A significant Mixture ingestion $X$ Sex interaction on $\mathrm{Nbm}$ amplitude and a significant Mixture ingestion X Sex X Hemisphere interaction on Pam latency were observed. ATD did not affect the MAEF source dipoles. The Pam latencies in both hemispheres had a significant negative relationship with the extent of ATD. The results suggest that the neurotransmitter serotonin is involved in early auditory cortical processing. Further, the serotonin modulation may be different in males and females.

[Neuropsychopharmacology 27:862-868, 2002] (C) 2002 American College of Neuropsychopharmacology. Published by Elsevier Science Inc.
KEY WORDS: Auditory; Event-related magnetic field; Magnetoencephalography (MEG); Middle-latency auditory magnetic field (MAEF); Serotonin; Tryptophan depletion

From the BioMag Laboratory, Engineering Centre, Helsinki University Central Hospital (SK, RJI), Cognitive Brain Research Unit, Department of Psychology, University of Helsinki (SK, JA, IPJ), Department of Pharmacology and Toxicology, University of Kuopio (SP, JL), Finland, and Massachusetts General Hospital-NMR Center, Harvard Medical School, Charlestown, MA, USA (IPJ).

Address correspondence to: Seppo Kähkönen, MD, Ph.D., BioMag Laboratory, Engineering Centre, Helsinki University Central Hospital, P.O. Box 340, FIN-00029 HUS, Finland. Tel.: +358 9 47175542; Fax: +358 9 47175781; E-mail: Seppo.Kahkonen@helsinki.fi

Received May 18, 2001; revised April 10, 2002; accepted April 20, 2002.

Online publication: $4 / 25 / 02$ at www.acnp.org/citations/ Npp042502292.
Low brain serotonin (5-HT) levels and function have been implicated in various psychopathologies including depression and anxiety. Acute tryptophan depletion (ATD) is a dietary intervention that rapidly lowers plasma tryptophan (Trp) and alters 5-HT synthesis, metabolism, and release in animals and humans (Young et al. 1985; Nishizawa et al. 1997; Stancampiano et al. 1997; Carpenter et al. 1998). It involves the administration of an amino acid mixture that lacks Trp, the amino acid precursor of serotonin. The effect of ATD on 5-HT synthesis and metabolism is twofold (Young et al. 1985). First, decline in plasma Trp occurs because the Trpdeficient amino acid mixture promotes synthesis of new proteins. Trp incorporated into protein comes from blood and tissue pools, and therefore its concentration in plasma and brain falls (Biggio et al. 1974; Gessa et al. 
1975). Second, all large neutral amino acids (LNAA) compete for the same carrier system that transport them across the blood-brain barrier (Oldendorf 1973). Brain Trp levels depend not only on availability of plasma Trp, but also on other LNAAs. Thus, amino acid competition for transport at the level of the blood-brain barrier plays a role in regulating brain Trp (Gessa et al. 1975). The result of ATD in rats is a decrease in brain Trp and 5-HT concentration (Moja et al. 1989) and a decrease of 5-HT and 5-hydroxyindolacetic acid (5-HIAA) concentrations in cerebrospinal fluid (Bel and Artigas 1996; Stancampiano et al. 1997).

Magnetoencephalography (MEG) is a method for the measurement of extracranial magnetic fields produced by ionic current flow generated mainly by cortical pyramidal cells. MEG is a non-invasive method for functional brain studies with temporal resolution as high as electroencephalography (EEG), but with more accurate source localization (Hämäläinen et al. 1993). Because of cortical folding in the Sylvian fissure, MEG is ideally suited for the localization and measurement of supratemporal auditory cortical activity (Hämäläinen et al. 1993).

Auditory evoked magnetic potentials (AEP) and fields (AEF), which allow the investigation underlying auditory processing, can be obtained by averaging EEG or MEG epochs time-locked to the presentation of auditory stimuli. The earliest cortical AEFs, the middlelatency AEFs (MAEFs), occur at about 20-70 ms from stimulus onset. The MAEFs are composed of several distinct deflections, with Pam peaking at approximately $30 \mathrm{~ms}$ and $\mathrm{Nbm}$ at about $45 \mathrm{~ms}$.

There is evidence to suggest that serotonergic activity may modulate auditory evoked potentials (Hegerl and Juckel 1993; Juckel et al. 1997; Dierks et al. 1999) and background EEG frequencies (Knott et al. 1999). The sound intensity dependence of auditory N1/P2 amplitude has been suggested to be a specific indicator of serotonin function (Hegerl and Juckel 1993; Juckel et al. 1997). Reduced serotonin function leads to higher intensity dependence of N1/P2 amplitude and vice versa. However, Dierks et al. (1999) were not able to confirm these findings using ATD in healthy subjects. In contract, this study showed that ATD decreases the intensity dependence of N1/P2 amplitudes. In the recent ATD study using a 52-g amino acid challenge, Hughes et al. (2000) found no changes in auditory N1, $\mathrm{P} 2$, or P3 amplitudes and latencies in men. Women are more susceptible to a lowering mood and to a greater memory impairment as a function of ATD (Ellenbogen et al. 1996; Riedel et al. 1999). The incidence of major depression, which is linked to serotonin deficiency, is higher in women than in men (Weissman and Olfson 1995).

We have previously shown that acetylcholine antagonist scopolamine enhances and dopamine D2-receptor antagonist haloperidol does not affect MAEF responses (Jääskeläinen et al. 1999; Kähkönen et al. 2001). Therefore, the present study investigated auditory processing after ATD by recording the Pam and Nbm components of MAEFs in healthy volunteers. The effect of gender on MAEF was also analyzed.

\section{METHODS}

\section{Subjects}

Fourteen subjects (aged 21-30 years; mean \pm SD, $24.1 \pm$ 3.3; seven females) participated in the study. An informed written consent and institutional ethical committee approval were obtained. Before the inclusion the subjects underwent a medical examination and laboratory tests of blood to exclude physical or mental health problems. They were also screened for mental problems by SCL-90 (Derogatis et al. 1973; Holi et al. 1998). The subjects reported having used no medications for two weeks prior to the study. The subjects were instructed to avoid alcohol for at least $48 \mathrm{~h}$, and caffeine for $12 \mathrm{~h}$ prior to the MEG recordings. All were non-smokers. The hearing level was confirmed by determining the auditory threshold with the auditory stimulator used for this experiment.

\section{Design}

Subjects were investigated after $5 \mathrm{~h}$ of ATD or control mixture ingestion by recording MAEF components Pam and $\mathrm{Nbm}$ in a randomized, double-blind, controlled cross-over design study. All subjects arrived at the laboratory at approximately 7:45 A.M. after an overnight fast. After a baseline heparinized blood sample was obtained for plasma total and free Trp, and for LNAA, the subject underwent either an ATD or control mixture administration. The amino-acid drink was composed of 15 amino acids with or without tryptophan, as described by Young et al. (1985). First, the subject was administered arginine, cysteine, and methionine, contained in capsules because of their very unpleasant taste. Then the subject consumed the remaining amino acids (i.e., alanine, glycine, histidine, isoleucine, leucine, lysine, phenylalanine, proline, serine, thereonine, tyrosine, and valine) as a chilled 300cc strawberry/mint flavored drink. The capsules and the drink were consumed during a period of 20-30 min. The second blood sample for measurements of plasma Trp and LNAA was taken after $5 \mathrm{~h}$ of ingestion of the mixture. The sessions were separated by one week.

\section{Stimulus Design and Data Acquisition}

During MEG recording, the subject sat in a comfortable chair in a magnetically and electrically shielded room 
(Euroshield, Eura, Finland). The auditory evoked fields were recorded with a 122-channel MEG device (Neuromag, Helsinki, Finland; Ahonen et al. 1993). Click stimuli (0.1-ms duration) were presented to the left ear at a rate of $10 \mathrm{~Hz}$ at $60 \mathrm{~dB}$ above an individually measured hearing threshold. A similar paradigm was used in our earlier psychopharmacological studies (Jääskeläinen et al. 1999; Kähkönen et al. 2001). During the stimulation, the subjects were instructed to concentrate on a video film of their own interest, and to ignore the clicks. Each two-channel sensor unit measured two independent magnetic field gradient components $\partial B_{z} / \partial x$ and $\partial B_{z} / \partial y$, the Z-axis being normal to the local helmet surface. The position of the subject's head relative to the recording instrument was determined by measuring the magnetic fields produced by marker coils in relation to cardinal points on the head (nasion, left and right pre-auricular points), determined before the experiment using an Isotrak 3D-digitizer (Polhemus, Colchester, VT) (Ahlfors and Ilmoniemi 1989). In addition, vertical and horizontal electro-oculograms (EOG) were recorded.

The recording passband was 1-250 $\mathrm{Hz}$ for MEG at a sampling rate of $1 \mathrm{kHz}$. Digital band-pass filtering was performed off-line at $10-120 \mathrm{~Hz}$ (Starr and Don 1988). The analysis period was $150 \mathrm{~ms}$ (including a $50-\mathrm{ms}$ prestimulus baseline). The first few responses and all the epochs coinciding with EOG or MEG changes exceeding $150 \mu \mathrm{V}$ or $3000 \mathrm{fT} / \mathrm{cm}$, respectively, were omitted from averaging. At least 1000 artifact-free responses were recorded and averaged.

\section{Determination of Plasma L-Trp and LNAAs}

Blood $(10 \mathrm{ml})$ was drawn from the left ulnar vein into a vacuum tube (Venoject, Belgium). The tube was cooled immediately and centrifuged (200 rev/min) for $10 \mathrm{~min}$. After centrifugation, the plasma was separated and stored at $-20^{\circ} \mathrm{C}$ until its assay for the amino acids. A modified procedure described by Qureshi et al. (1984) was used in plasma assay for amino acids. Coefficients of variation of $1.2,1.05,1.11,1.14,5.5$, and $1.0 \%$ were determined for tryphophan, tyrosine, valine, phenylalanine, isoleucine and leucine (at $150 \mathrm{pmol} / \mu \mathrm{l}$ ), respectively. The detection limit was $10 \mathrm{pmol} / \mu \mathrm{l}$.

\section{Data Analysis}

The distinct AEF peaks were obtained from latency ranges of 25 to $40 \mathrm{~ms}$ for Pam and 40 to $55 \mathrm{~ms}$ for $\mathrm{Nbm}$. The AEF peak latencies and amplitudes were measured from the channel pair showing the highest-amplitude responses (the amplitude being determined as $|a|=$ $\left.\left(\left(\partial B_{z} / \partial x\right)^{2}+\left(\partial B_{z} / \partial y\right)^{2}\right)^{1 / 2}\right)$, separately for the left and right temporal areas. The AEF sources were located by a least-squares fit of a single equivalent current dipole (ECD) using a fixed subset of 34 channels separately over each auditory cortex (Hämäläinen et al. 1993). Dipole fits with at most $30 \%$ residual variance were considered successful. The statistical analysis was carried out with 3-factor (Mixture X Hemisphere X Sex) repeatedmeasures ANOVAs. Paired and unpaired $t$-tests were used for a priori comparison when appropriate. The extent of the tryptophan depletion was correlated with MAEF responses using Spearman correlation.

\section{RESULTS}

Blood samples showed that the Trp manipulation was successful in all subjects. ATD lowered the total plasma Trp levels by $75 \%$, free Trp level by $47 \%$, and the ratio Trp/ $\Sigma$ LNAA by $92 \%$. The control mixture increased total Trp level by $45 \%$ and free $\operatorname{Trp}$ by $32 \%$, and decreased the ratio $\operatorname{Trp} / \Sigma$ LNAA by $35 \%$. The ratio tyrosine/ $\Sigma$ LNAA did not differ between ATD and control experiment (Table 1). Click stimulation elicited prominent Pam and $\mathrm{Nbm}$ responses (Figure 1).

Table 2 shows the peak latencies and peak amplitudes of the MAEF components in ATD and control conditions over both measured hemispheres. ATD resulted in a significant main effect on Pam latencies and a near-significant main effect on Pam amplitudes $\left(\mathrm{F}_{1,6}=\right.$ $5.70, p=.05 ; \mathrm{F}_{1,6}=5.27, p=.06$, respectively). A significant Mixture ingestion $X$ Sex interaction on Nbm amplitude $\left(\mathrm{F}_{1,6}=6.32, p=.04\right)$ and a significant Mixture ingestion $X$ Sex $X$ Hemisphere interaction on Pam latency $\left(\mathrm{F}_{1,6}=7.14, p=.04\right)$ were observed. The dipole moments of Pam and Nbm were not changed after ATD administration over either hemisphere. The source locations of the responses were not significantly influenced by ATD (Table 3). The latencies of Pam at the contralateral and ipsilateral hemispheres correlated negatively with $\operatorname{Trp} / \Sigma$ LNAA at ATD $(\sigma=-0.64 ; p=.01$, and $\sigma=$ $-0.60 ; p=.02$, respectively).

Table 1. Amino Acid Levels (pmol/ $\mu \mathrm{l})$ and Uptake Ratios in Different Conditions

\begin{tabular}{lccc}
\hline Amino acids & Time (h) & Trp depletion & Control experiment \\
\hline Total Trp & 0 & $62.5 \pm 13.1$ & $60.9 \pm 26.3$ \\
& 5 & $15.6 \pm 4.2$ & $100 \pm 25.1$ \\
Free Trp & 0 & $5.8 \pm 2.0$ & $7.0 \pm 3.2$ \\
& 5 & $3.1 \pm 1.8$ & $9.2 \pm 4.3$ \\
Ratio & 0 & $15.6 \pm 5.3$ & $12.3 \pm 2.8$ \\
$\quad$ LLNAA/Trp & 5 & $1.3 \pm 0.7$ & $8.0 \pm 2.1$ \\
Ratio & 0 & $10.5 \pm 2.8$ & $10.6 \pm 1.7$ \\
$\quad$ LLNAA/Tyr & 5 & $4.6 \pm 1.5$ & $5.0 \pm 1.8$ \\
\hline
\end{tabular}

Trp = Tryptophan; LNAA $=$ Large neutral amino acid; Tyr $=$ Tyrosine.

Baseline Total Trp, Free Trp, Ratio LLNAA/Trp, and Ratio $\Sigma$ LNAA/ Tyr did not differ between Trp depletion and control experiment (paired $t$-test; $p>0.05$ ). 


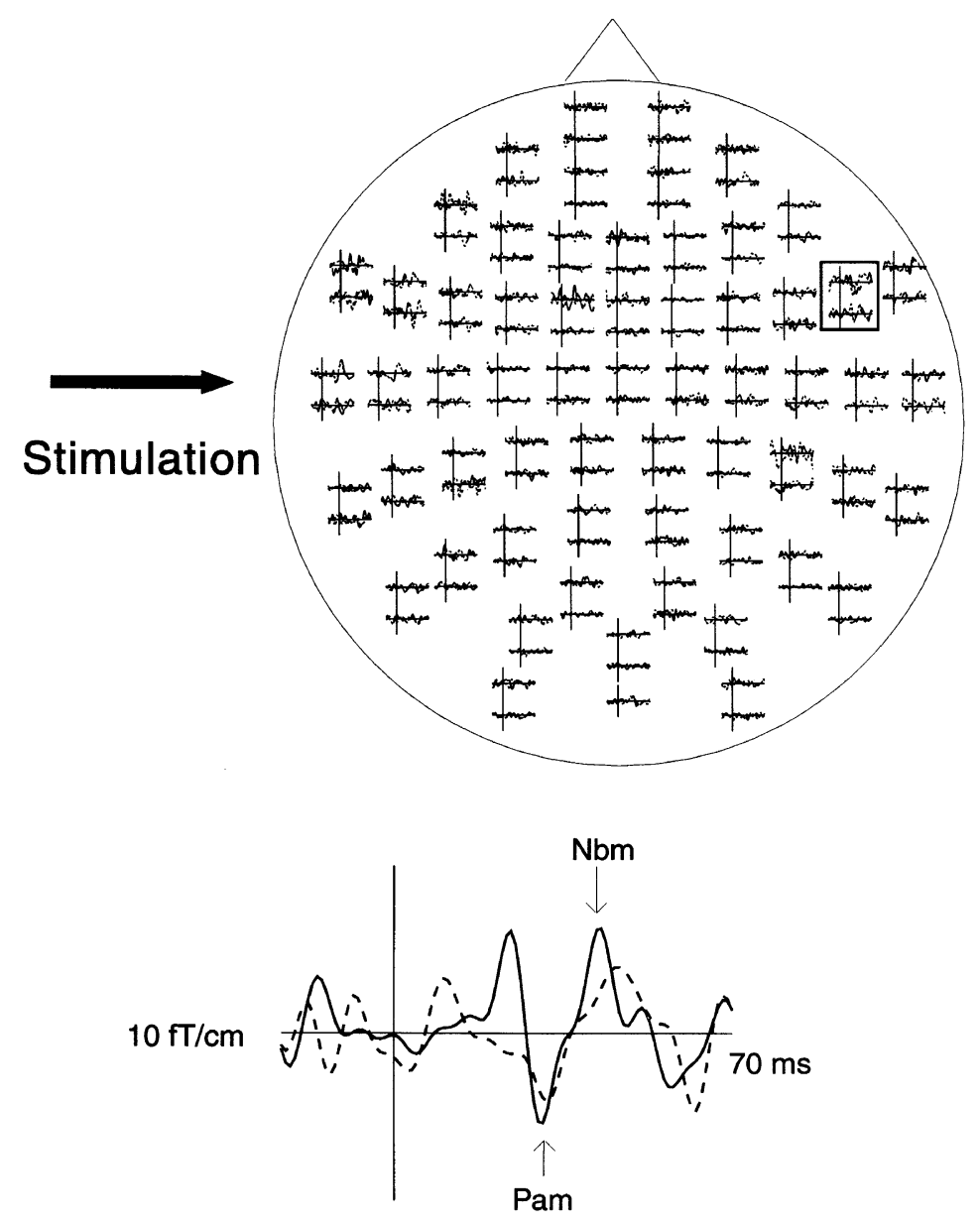

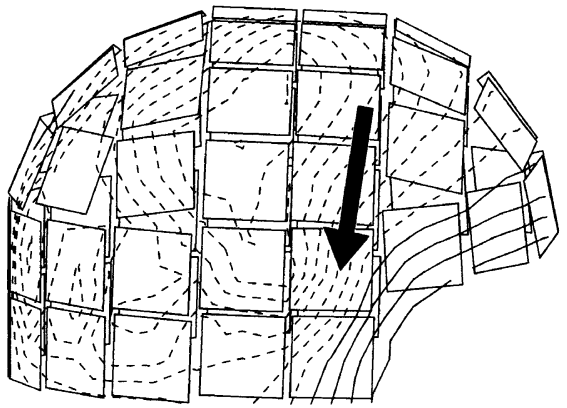

Control experiment

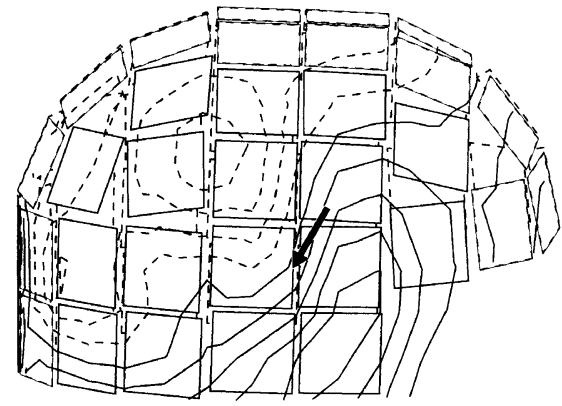

Tryptophan depletion

Figure 1. Upper panel. MAEF response in one subject measured with magnetometer during tryptophan depletion (dotted lines) and control experiment (solid line). The stimuli were delivered to the left ear. Middle panel. The enlarged response presents the channel showing largest responses over the right temporal areas (contralateral to the ear stimulated). Lower diagrams. Contour maps at the peak of $\mathrm{Nbm}$. Solid lines indicate magnetic flux lines where it enters outside the head and dotted lines areas indicate magnetic flux lines exits within the head. Contour line separation is $3 \mathrm{fT}$. The arrows depict the strengths (proportional to arrow length) and locations of single equivalent current dipoles of Nbm applied to data.

\section{DISCUSSION}

In our experiment, ATD caused significant reduction in

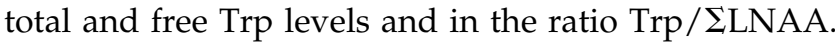

The level of reduction of amino acids is in line with studies with ATD performed earlier. A recent positron emission tomography (PET) study indicated that ATD markedly decreases serotonin synthesis in different 
Table 2. Peak Latencies and Amplitudes of MAEF Components after Tryptophan Depletion and Control Experiment

\begin{tabular}{lllrr}
\hline Deflection & Hemisphere & \multicolumn{1}{c}{ Response } & Tryptophan Depletion & Control Experiment \\
\hline Pam & Contralateral & Latency $(\mathrm{ms})$ & $34.6 \pm 4.0$ & $32.0 \pm 3.9$ \\
Pam & Ipsilateral & Latency $(\mathrm{ms})$ & $32.0 \pm 3.9$ & $30.4 \pm 3.6$ \\
Pam & Contralateral & Amplitude $(\mathrm{fT} / \mathrm{cm})$ & $5.6 \pm 2.3$ & $5.7 \pm 2.5$ \\
Pam & Ipsilateral & Amplitude $(\mathrm{fT} / \mathrm{cm})$ & $5.0 \pm 2.3$ & $5.2 \pm 3.2$ \\
$\mathrm{Nbm}$ & Contralateral & Latency $(\mathrm{ms})$ & $50.1 \pm 5.8$ & $47.5 \pm 4.8$ \\
$\mathrm{Nbm}$ & Ipsilateral & Latency $(\mathrm{ms})$ & $49.5 \pm 4.8$ & $47.8 \pm 3.8$ \\
$\mathrm{Nbm}$ & Contralateral & Amplitude $(\mathrm{fT} / \mathrm{cm})$ & $5.4 \pm 2.4$ & $5.1 \pm 2.1$ \\
$\mathrm{Nbm}$ & Ipsilateral & Amplitude $(\mathrm{fT} / \mathrm{cm})$ & $6.0 \pm 1.5$ & $5.6 \pm 2.0$ \\
\hline
\end{tabular}

brain regions (Nishizawa et al. 1997). Furthermore ATD has been shown to decrease the concentration of 5-HIAA, the principal metabolite of serotonin in cerebrospinal fluid in normal volunteers, by over 30\% (Carpenter et al. 1998; Williams et al. 1999). There is evidence from animal and human studies that an amino acid drink deficient in tyrosine (Tyr) can interfere with central catecholamine function in a way similar to the effects of ATD in humans (Moja et al. 1996; Harmer et al. 2001). The ATD and control experiment influenced the ratio Tyr/ LLNAA in a similar manner indicating that metabolism of dopamine and noradrenaline were not affected in our experiment. Based on these consistent findings, one may expect that ATD reduced cerebral serotonin synthesis in the present subjects as well.

Table 3. Mean \pm SD Dipole Positions in Cartesian Coordinates, Dipole Moments (Q), and Percentage of Sources Successfully Modeled in Tryptophan Depletion and Control Conditions

\begin{tabular}{llcc}
\hline ERF Deflection & & $\begin{array}{c}\text { Tryptophan } \\
\text { Depletion }\end{array}$ & $\begin{array}{c}\text { Control } \\
\text { Experiment }\end{array}$ \\
\hline Pam (contralateral) & $x(\mathrm{~mm})$ & $50 \pm 18$ & $53 \pm 19$ \\
& $y(\mathrm{~mm})$ & $14 \pm 16$ & $19 \pm 15$ \\
& $z(\mathrm{~mm})$ & $73 \pm 14$ & $66 \pm 19$ \\
& $Q$ (nAm) & $2.8 \pm 3.1$ & $2.1 \pm 1.5$ \\
& $\%$ Modeled & 72 & 57 \\
Pam (ipsilateral) & $x(\mathrm{~mm})$ & $-42 \pm 12$ & $-38 \pm 10$ \\
& $y(\mathrm{~mm})$ & $9 \pm 12$ & $10 \pm 13$ \\
& $z$ (mm) & $57 \pm 10$ & $64 \pm 22$ \\
& $Q$ (nAm) & $4.4 \pm 3.3$ & $4.3 \pm 1.2$ \\
& $\%$ Modeled & 50 & 43 \\
Nbm (contralateral) & $x$ (mm) & $39 \pm 12$ & $34 \pm 36$ \\
& $y$ (mm) & $6 \pm 12$ & $9 \pm 12$ \\
& $z$ (mm) & $64 \pm 10$ & $67 \pm 25$ \\
& $Q$ (nAm) & $4.2 \pm 1.9$ & $2.7 \pm 2.0$ \\
& $\%$ Modeled & 58 & 58 \\
& $x$ (mm) & $-45 \pm 24$ & $-29 \pm 33$ \\
& $y$ (mm) & $7 \pm 19$ & $7 \pm 25$ \\
& $z$ (mm) & $41 \pm 32$ & $61 \pm 20$ \\
& $Q$ (nAm) & $3.1 \pm 1.8$ & $2.7 \pm 1.0$ \\
& $\%$ Modeled & 36 & 64 \\
& & &
\end{tabular}

The $x$-axis runs from left pre-auricular point to the right. The $y$-axis runs through the nasion, and $z$-axis points upwards. Unpaired $t$-test; $p>.05$
Our results showed that ATD significantly modulated MAEF responses. Results from both MEG and intracranial recordings have suggested that MAEFs are generated in the superior aspects of the temporal lobes, in or near the primary auditory cortex (Liegois-Chauvel et al. 1994; Scherg et al. 1989). Notably, the densest serotonergic innervation has been found in the superior temporal gyrus, including auditory cortices, with higher density in the primary than in the secondary auditory cortex (Azmitia and Gannon 1986; Lewis et al. 1986; Palmer et al. 1987). Within the primary auditory cortex, layer IV, which receives the most of specific thalamic sensory input (Zilles 1990; Pandya and Rosene 1993) appears to be also most densely innervated by serotonin (Lewis et al. 1986; Campell et al. 1987). Serotonergic projections are suggested to modulate the early phases of sensory processing at the cortex (Morrison et al. 1982). Furthermore, ATD causes the reduction of serotonin synthesis at the temporal cortex (Nishizawa et al. 1997). It is likely that in our study the ATD caused a reduction of serotonin synthesis in the temporal cortex, which is shown to modulate the MAEF responses. Thus, our MEG results suggest that serotonin transmission modulates the early phase of cortical auditory processing in the human brain.

Several clinical and experimental studies using EEG have suggested that AEPs could be modulated by serotonin (Hegerl and Juckel 1993; Juckel et al. 1997; Dierks et al. 1999). EEG detects particularly the radial currents generated by activity in the cortical gyri, but also the tangential ones in the sulci. Middle-latency auditory responses have been shown to be generated by tangential sources in the auditory cortex, but also by radial ones (Mäkelä et al. 1994; Scherg and von Cramon 1986, 1989). Signals from deep sources, such as the postulated subcortical sources affected by ATD contributing to the MAEP (Woods et al. 1987; Nishizawa et al. 1997), can be detected by EEG. In contrast to EEG, MEG is selectively sensitive to tangential cortical but insensitive to subcortical sources of activity (Hämäläinen et al. 1993). This is due to the fact that the magnetic fields fall off rapidly with increasing distance from the source. Both in EEG and MEG, source localization requires modeling. The superiority of MEG to EEG is largely based on MEG be- 
ing practically unaffected by the detailed structure and conductivity of the skull and scalp. Furthermore, MEG is selectively sensitive to tangential source currents in temporal cortex, which make the source modeling more stable than in EEG (Hämäläinen et al. 1993). Hence, it is possible that changes in electromagnetic activity following ATD administration is not detected by MEG in the gyri of the temporal cortex. In future studies, a combined use of MEG and EEG will provide a more complete view of effects of ATD in healthy volunteers and in the clinical population.

Our results are in line with the earlier findings that MAEF responses after ATD are different in women compared with men. The rate of serotonin metabolism is higher in females than in males (Young et al. 1980; Agren et al. 1986). In the PET study of Nishizawa et al. (1997), the reduction of serotonin synthesis in the temporal cortex following ATD was much higher in women than in men. The difference in the rate of serotonin synthesis after ATD may partly explain the different MAEF responses in different sexes. However, our results are based on a small number of subjects. Further studies with a larger number of subjects are needed to confirm or reject this result.

Previously, only a few attempts have been made to elucidate the transmitters involved in middle-latency evoked-response generation. Our findings (Jääskeläinen et al. 1999) and those of Buchwald et al. (1991) have suggested that brain acetylcholine plays an important role in the generation of the Pa response. In these studies, the $\mathrm{Pa} / \mathrm{Pam}$ amplitude was enhanced by muscarine receptor antagonist scopolamine, and was reduced by the agonist physostigmine. We recently studied the effects of dopamine D2 receptor antagonist haloperidol on MAEF responses. No differences were observed in latencies, amplitudes, dipole moments or locations of MAEF responses between haloperidol and placebo (Kähkönen et al. 2001). The results of the later and the present study indicate that different amine neurotransmitters, serotonin and dopamine, have differential effects on MAEF generation.

In conclusion, our novel MEG results, demonstrating ATD-induced modulation of MAEF responses, suggest that serotonin modulates MAEF generation in the primary auditory cortex and thus may play a role in early cortical processing. The modulation of serotonin effects on early cortical processing may be different in females compared with males. The findings might also be associated with auditory processing deficits observed in psychiatric and neurological disorders with serotonin deficiencies.

\section{ACKNOWLEDGMENTS}

Presented in part at the seventh international conference on Functional Mapping of the Human Brain. June 10-14, 2001, Brighton, Great Britain.
This work was supported by the Helsinki University Central Hospital Research Funds and the Academy of Finland. We thank Mrs. Suvi Heikkilä and Mr. Teemu Peltonen for their excellent technical assistance. Thanks are due to Mr. Christopher Bailey for his language revision.

\section{REFERENCES}

Agren H, Mefford IN, Rudorfer MV, Linnoila M, Potter WZJ (1986): Interacting neurotransmitter systems. A nonexperimental approach to the 5HIAA-HVA correlation in human CSF. Psychiatr Res 20:175-193

Ahlfors S, Ilmoniemi RJ (1989): Magnetometer position indicator for multichannel MEG. In Williamson SJ, Hoke M, Romani GL (eds), Advances in Biomagnetism. New York, Plenum Press, pp 673-676

Ahonen AI, Hämäläinen MS, Kajola MJ, Knuutila JE, Laine PP, Lounasmaa OV, Parkkonen LT, Simola JT, Tesche CD (1993): 122-channel SQUID instrument for investigating the magnetic signals from the human brain. Physica Scripta T49:198-205

Azmitia EC, Gannon PJ (1986): The primate serotonergic system: a review of human and animal studies and a report on macaca fasicularis. Adv Neurol 43:407-468

Bel N, Artigas F (1996): Reduction of serotonergic function in rat brain by tryptophan depletion: effects in control and fluvoxamine-treated rats. J Neurochem 67:669-676

Biggio G, Fadda F, Fanni P, Tagliamonte A, Gessa GL (1974): Rapid depletion of serum tryptophan, brain tryptophan, serotonin and 5-hydroxyindoleacetic acid by a tryptophan-free diet. Life Sci 14:1321-1329

Buchwald JS, Rubinstein EH, Schwafel J, Strandburg RJ (1991): Mid-latency evoked responses: differential effects of a cholinergic agonist and antagonist. Electroen Clin Neuro 80:303-309

Campell MJ, Lewis DA, Foote SL, Morrison JH (1987): Distribution of choline acetyltransferase-, serotonin-, dopamine-b-hydroxylase-, tyrosine hydroxylase-immunoreactive fibers in monkey primary auditory cortex. J Comp Neurol 261:209-220

Carpenter I, Anderson G, Pelton G, Gudin J, Kirwin P, Price L, Heninger G, McDougle C (1998): Tryptophan depletion during continuous CSF sampling in healthy human subjects. Neuropsychopharmacology 19:26-35

Derogatis LR, Lipman RS, Covi L (1973): SCL-90: an outpatient psychiatric rating scale- preliminary report. Psychopharmacology 9:13-28

Dierks T, Barta S, Demisch L, Schmeck K, Englert E, Kewitz A, Maurer K, Poustka F (1999): Intensity dependence of auditory evoked potentials (AEPs) as biological marker for cerebral serotonin levels: effects of tryptophan depletion in healthy subjects. Psychopharmacology 146:101-107

Ellenbogen MA, Young SN, Dean P, Palmour RM, Benkelfat C (1996): Mood response to acute tryptophan depletion in healthy volunteers: sex differences and temporal stability. Neuropsychopharmacol 15:465-474

Hämäläinen M, Hari R, Ilmoniemi RJ, Knuutila J, Lounasmaa OV (1993): Magnetoencephalography - theory, instrumentation, and application to noninvasive studies of the working human brain. Rev Mod Phys 65:413-498 
Harmer CJ, Mc Tavish SFB, Clark L, Goodwin GM, Cowen PJ (2001): Tyrosin depletion attenuates dopamine function in healthy volunteers. Psychopharmacology (Berl) 154:105-111

Hegerl U, Juckel G (1993): Intensity dependence of auditory evoked potentials as an indicator of central serotonergic neurotransmission: a new hypothesis. Biol Psychiatry 33:173-187

Holi MM, Sammanlahti PR, Aalberg VA (1998): Finnish validation study of the SCL-90. Acta Psychiatr Scand 97:42-46

Hughes JH, Ashton CH, Matthews D, Young AH (2000): Acute depletion of plasma tryotophan does not alter electrophysiological variables in healthy males. Psychopharmacology (Berl) 152:119-121

Gessa G, Biggio G, Fadda F, Corsini G, Tagliamonte A (1975): Tryptophan-free diet: a new means for rapidly decreasing brain tryptophan content and serotonin synthesis. Acta Vitaminol Enzymol 29:72-78

Jääskeläinen IP, Hirvonen J, Huttunen J, Kaakkola S, Pekkonen E (1999): Scopolamine enhances middlelatency evoked magnetic fields. Neurosci Lett 259:41-44

Juckel G, Molnar M, Hegerl U, Csepe V, Karmos G (1997): Auditory-evoked potentials as indicator of brain serotonergic activity-first evidence in behaving cats. Biol Psychiatry 41:1181-1195

Kähkönen S, Ahveninen J, Pekkonen E, Kaakkola S, Huttunen J, Peltonen $\mathrm{T}$, Ilmoniemi RJ, Jääskeläinen IP (2001): Early cortical auditory processing appears to be independent of dopamine D2 receptor modulation as measured by whole-head magnetoencephalography. Psychiat Res Neuroimaging 25:498-504

Knott VJ, Howson AL, Perugini M, Ravindran AV, Young SN (1999): The effect of acute tryptophan depletion and fenfluramine on quantitative EEG and mood in healthy male subjects. Biol Psychiat 46:229-238

Lewis DA, Campbell MJ, Foote SL, Morrison JH (1986): The monoaminergic innervation of primate neocortex. Human Neurobiol 5:181-188

Liegois-Chauvel C, Musolino A, Badier JM, Marquis P, Chavel P (1994): Evoked potentials recorded from the auditory cortex in man: evaluation and topography of the middlelatency components. Electroen Clin Neuro 92:204-214

Mäkelä JP, Hämäläinen M, Hari R, McEvoy L (1994): Wholehead mapping of middle-latency evoked magnetic fields. Electroen Clin Neuro 92:414-421

Moja EA, Cipolla P, Castoldi D, Tofanetti O (1989): Doseresponse decrease in plasma tryptophan and in brain tryptophan and serotonin after tryptophan-free amino acid mixtures in rats. Life Sci 44:971-976

Moja EA, Lucini V, Benedetti F, Lucca A (1996): Decrease in plasma phenylalanine and tyrosine after phenylalaninetyrosine free amino acid solutions in man. Life Sci 58:2389-2395

Morrison JH, Foote SL, Molliver ME, Bloom FE, Lidov HGW (1982): Noradrenergic and serotonergic fibers innervate complementary layers in monkey primary visual cortex: an immunohistochemical study. Proc Natl Acad Sci USA 79:2401-2405

Nishizawa S, Benkelfat C, Young SN, Leyton M, Mzengeza S, de Montigny C, Blier P, Diksic M (1997): Differences between males and females in rates of serotonin synthesis in human brain. Proc Natl Acad Sci USA 94:53085313

Oldendorf W (1973): Stereospecificity of blood-brain barrier permeability to amino acids. Am J Physiol 224:967-969

Palmer AM, Wilcock GK, Esiri MM, Francis PT, Bowen DM (1987): Monoaminergic innervation of the frontal and temporal lobes in Alzheimer's disease. Brain Res 401:231-238

Pandya DN, Rosene DL (1993): Laminar termination patters of thalamic, callosal, and association afferens in the primary auditory area of the rhesus monkey. Exp Neurol 119:220-234

Qureshi GA, Fohlin L, Bergström J (1984): Application of high-performance liquid chromatography to the determination of tree amino acids in physiological fluids. J Chromatogr 297:91-100

Riedel WJ, Klaassen T, Deutz NE, van Someren A, van Praag HM (1999): Tryptophan depletion in normal volunteers produces selective impairment in memory consolidation. Psychopharmacology (Berl) 141:362-369

Scherg M, von Cramon D (1986): Evoked dipole source potentials of the human auditory cortex. Electroen Clin Neuro 65:344-360

Scherg M, Hari R, Hämäläinen M (1989): Frequency-specific sources of the auditory N19-P30-P50 response detected by a multiple source analysis of evoked magnetic fields and potentials. In Williamson SJ, Hoke M, Stroink G, Kotani M (eds), Advances in Biomagnetism. New York, Plenum Press, pp 97-100

Stancampiano R, Melis F, Sarais L, Cocco S, Cugusi C, Fadda F (1997): Acute administration of a tryptophan-free amino acid mixture decreases 5-HT release in rat hippocampus in vivo. Am J Physiol 272:R991-R994

Starr A, Don M (1988): Brain potentials evoked by auditory stimuli. In Picton TW (ed), Handbook of Electroencephalography and Clinical Neurophysiology. Amsterdam, Elsevier, pp 97-157

Zilles K (1990): Cortex. In Paxinos G (ed), The Human Nervous System. San Diego, Academic Press, pp 757-802

Young SN, Smith SE, Pihl RO, Ervin FR (1985): Tryptophan depletion causes a rapid lowering of mood in normal males. Psychopharmacology 87:173-177

Young SN, Gauthier S, Anderson GM, Purdy WC (1980): Tryptophan, 5-hydroxyindoleacetic acid and indoleacetic acid in human cerebrospinal fluid: interrelationships and the influence of age, sex, epilepsy and anticonvulsant drugs. J Neurol Neurosurg Psychiat 43:438-445

Weissman MM, Olfson M (1995): Depression in women: implications for health care research. Science 269:799-801

Williams WA, Shoaf SE, Hommer D, Rawlings R, Linnoila M (1999): Effects of acute tryptophan depletion on plasma and cerebrospinal fluid tryptophan and 5-hydroxyindolacetic acid in normal volunteers. J Neurochem 72:1641-1647

Woods DL, Clayworth CC, Knight RT, Simpson GV, Naeser MA (1987): Generators of middle- and long-latency auditory evoked potentials: implications from studies with bitemporal lesions. Electroen Clin Neuro 68:132-148 